and methods of measurement were similar to those used before by two of the authors. ${ }^{1}$

A potassium hydride surface illuminated by an incandescent lamp gave currents ample for measurement of the principle ionization potential and potentials of inelastic impact. Consistent initial potential corrections were obtained from measurement of the potential at which the total current started. The ionization potential thus corrected was I6.0 volts. Inelastic collisions occurred at 10.5 and 22.3 volts.

The photo-electric cathode was replaced by a Wehnelt cathode. The results obtained were practically identical with the above at the same pressures. With higher pressures inelastic impacts occurred at 10.5, 16 and 21 volts. In this respect the results are entirely consistent with the work of Mohler and Foote.

As the above method of applying initial corrections may be questionable, measurements were made with mercury vapor as well as hydrogen in the tube. Pressures were adjusted to give nearly equal amounts of ionization from the two gases and the difference between ionization points was measured. The resulting value for hydrogen is 15.5 volts.

We conclude from the above results that the dissociation of hydrogen by an incandescent wire plays no appreciable part in the phenomena observed. The potentials of inelastic collision at 10.5 and 22.3 indicate resonance potentials at 10.5 and Ir. 8 volts, due to collisions with normal hydrogen. The possibility that 22.3 volts is a resonance potential and not due to successive impacts is not excluded by this work.

Bureau of Standards.

\title{
Photo-electric Phenomena in Coated-filament Audion Bulbs.
}

By R. C. Gibis and Edna L. Meacham.

$\mathrm{CASE}^{2}$ and Merritt $^{3}$ have recorded certain observations made upon the photoactive behavior of coated-filament vacuum tubes. Additional experiments intended to examine in more detail some phases of their work have been performed.

With one of the tubes previously used by Merritt, study was mainly made upon the increase in thermionic current that occurs when the coated filament is illuminated. This increase will be referred to as the photo-electric current. For various voltages applied between filament and plate the photo-electric current was found to increase with increase of filament current in very much the same way as the thermionic current. The size of the photo-electric current depended upon the potential of the grid. In order to eliminate a steady drift in thermionic current which was in one direction when the grid was connected to the plate and in the opposite direction when the grid was

1 Bureau of Standards, Sci. Paper No. 400.

2 Phys. Rev., Vol. XVII., p. 398, I92 I.

3 Phys. Rev., Vol. XVII., p. 525, 1921. 
connected to the filament, a high resistance was connected between filament and plate and the grid connected so as to maintain it at a potential between that of filament and plate. With this connection the photo-electric current reached values as large as $24 \times 10^{-8}$ amperes for a filament current of 0.9 ampere when it was illuminated by a 400 -watt incandescent lamp operated on a storage battery and placed about one foot from the filament of the tube, a narrow slit being interposed to prevent illumination of other parts of the tube. With the grid floating a maximum for the photo-electric current was found for various plate potentials at a filament temperature corresponding to a filament current of about 0.83 ampere. For constant filament current the photo-electric current showed a saturation effect at the higher plate potentials.

It was found that from 8 to ro minutes were required for the photo-electric current to reach its maximum after turning the illumination on the filament. Furthermore the decay of this current after the illumination was turned off was rather slow. The time of decay varied from 25 to 50 minutes depending upon various conditions of plate potential, filament current, and previous treatment of filament.

The ratio of photo-electric current to thermionic current for the same filament temperature decreased with increase in filament current, with an irregularity that of ten showed a distinct minimum and maximum between 0.5 and 0.6 ampere in the filament. This irregularity may be due to the fact that the filament was not brought to the same initial condition before the determination of each ratio.

It was early discovered that the ratio just mentioned depended, for a given filament temperature, to a very great extent upon the temperature and light treatment of the filament just previous to its determination.

A long series of observations were made at one filament temperature to determine the effect of this previous temperature and light treatment. At the filament temperature used, that corresponding to a filament current of 0.5 ampere, the ratio of photo-electric current to thermionic current was much larger when the filament had been illuminated at a higher temperature for some time just before. Previous illumination at a lower temperature reduced the ratio. If the filament were not illuminated during these higher and lower temperature treatments the ratio in each case was larger than when illuminated, the difference being more marked for the lower temperature treatment.

Cornell University, August, I92I.

The Information Service of the National Research Council.

By Robert M. Yerkes.

Chairman, Research Information Service.

THE clearing-house for science and technology, established by the National Research Council in I919, has developed rapidly. It now contains uniquely valuable informational apparatus whose serviceability to investigators will increase as it is perfected and utilized in answering requests. 